\title{
Do peak/slack seasons influence semiconductor machine outliers? A back propagation neural network analysis
}

\author{
Keng-Chieh Yang ${ }^{1, a}$, Chia-Hui Huang ${ }^{2}$ and Po-Hong Shih ${ }^{3}$ \\ ${ }^{1}$ Department of Information Management, Hwa Hsia University of Technology, New Taipei City 235, Taiwan \\ ${ }^{2}$ Department of Business Administration, National Taipei University of Business, Taipei City 100, Taiwan \\ ${ }^{3}$ Institute of Information Management, National Chiao Tung University, Hsinchu 300, Taiwan
}

\begin{abstract}
The semiconductor industry is often affected by the economy impact, which also influences the production schedule planning. The back propagation neural network model has the advantages of great precision and effectiveness. This research uses Novellus Vector Machine and its Remote Process Controller (RPC) function to collect the data. This study detects the gas transmission pressure of chamber. We uses fault detection and classification (FDC) to analyze the model. FDC can detect the deviations of the machine parameters when the parameters deviate from the original value and exceed the range of the specification. This study adopts back propagation neural network model and gray relational analysis as tools to analyze the data and detect the semiconductor machine outliers. The findings indicate that peak seasons have less outliers than slack seasons.
\end{abstract}

\section{Introduction}

Semiconductor industry often faces peak seasons and slack ones and this may impact the manufacturing and business operation. Generally the first quarter is the slack season, and the third quarter is the peak season [10]. The iSuppli research report also pointed out that the revenues of semiconductor industry in the first quarter is usually lower than that of the third or fourth quarter in the previous year. In the first quarter the number of orders is also less than that of the fourth quarter in the previous year. The utilization of foundry equipment is also put baptism of fire. Slack season will make the equipment and person idle $[13,18]$. The impact of idle equipment will influence the yield rate in the future. This research tries to study the yield rate of equipment in the peak seasons and slack ones, especially when equipment is shut down and restarted.

\section{Methods and materials}

Manufacturing process quality control is a set of procedures intended to ensure that a product or service adheres to a defined set of quality criteria or meets the requirements of the client [11]. In this section, we introduce quality control methods in the semiconductor wafer manufacturing process. Our study introduces the basic concepts of FDC and back propagation neural network in the Semiconductor Industry.

\footnotetext{
${ }^{\mathrm{a}}$ Corresponding author : andesyoung.tw@gmail.com
} 


\subsection{Quality control in semiconductor manufacturing}

The semiconductor manufacturing factory is usually using SPC to detect the quality of real-time processes. These information are often used to adjust the machine parameters after the event. SPC control charts are important tools to diagnose abnormalities of process [5,16]. Using these charts, engineers can understand the quality of the wafer. If the output of numerical data are between the upper bound (UCL) and lower bound (LCL), it means the quality is qualified. Otherwise, the quality is flaw.

Control charts are one of the most commonly used methods of Statistical Process Control (SPC), which monitors the stability of a process [17]. The main functions of a control chart include the data points, a centerline (mean value), and upper and lower limits (bounds to indicate where a process output is considered "out of control") [5,8]. They visually display the fluctuations of a particular process variable, such as gas pressure, in a way that lets the engineer easily determines whether these variations fall within the specified process limits.

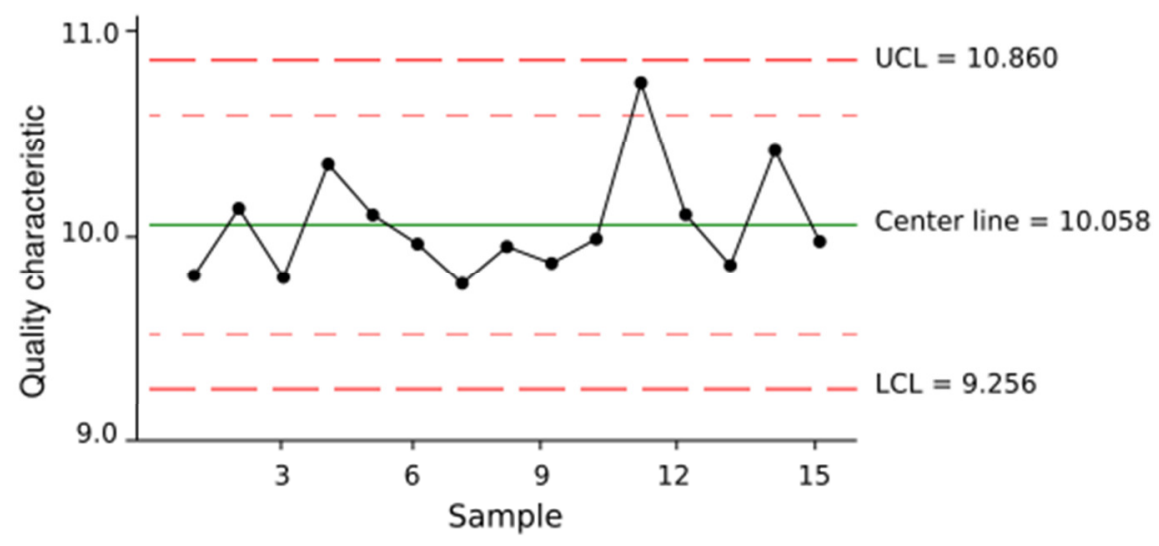

Figure 1. Sample of SPC control chart.

Fault Detection and Classification (FDC) contains two functions: fault detection (FD), and the fault classification (FC). Engineers are based on results of FD test to take some necessary actions. Different fault requires different corrective actions, while FC function is classified fault based on statistics Eigen-values so that engineers can quickly refer to the machine error code and restore the machine to normal state within the most minimum time [9].

In the semiconductor wafer process, when the machine produces a certain number of wafer, some parameters will drift from original ones. So, at this moment, FDC can detect deviations within a short time. When the parameters deviate from the original value, and may be beyond the range of the set interval, this needs to apply the Run-to-Run adjustments to modify the parameters directly and continuously collecting the running parameters of the machine and constant feedbacks [1]. Based on the previous activities of quality control, engineers can adjust the machine parameters to ensure that the production are within normal operations [15-16].

Engineers use FDC monitor to ensure the information of production status correct, including the manufacturing process, machine operating conditions, parameters, and use of the recipe. Engineers must check the machine status before the operation in issues; otherwise, when the production is finished, it would lead to the business loss. FDC monitor can avoid the waste of production capacity, reduce failures and ensure the producing yield increases [1, 19].

\subsection{Back propagation neural network}

The back propagation (BP) neural network algorithm is a multi-layer feed forward network trained according to error back propagation algorithm and is one of the most widely applied neural network models $[2,6]$. BP network can be used to learn and store a great deal of mapping relations of input- 
output model, and no need to disclose in advance the mathematical equation that describes these mapping relations. Its learning rule is to adopt the steepest descent method in which the back propagation is used to regulate the weighted value and threshold value of the network to achieve the minimum error sum of square $[12,14]$. This paper focuses on the analysis of the characteristics and mathematical theory of BP neural network and also points out the shortcomings of BP algorithm as well as several methods for improvement.

$\mathrm{BP}$ algorithm is a method to monitor learning. It utilizes the methods of mean square error and gradient descent to realize the modification to the connection weight of network [7]. The modification to the connection weight of network is aimed at achieving the minimum error sum of squares $[4,6]$. In this algorithm, a little value is given to the connection value of network first, and then, a training sample is selected to calculate gradient of error relative to this sample.

The BP learning process can be described as follows [3]:

(1) Forward propagation of operating signal: the input signal is propagated from the input layer, via the hidden layer, to the output layer. During the forward propagation of operating signal, the weighted value and offset value of the network are maintained constant and the status of each layer of neuron will only exert an effect on that of next layer of neuron. In case that the expected output cannot be achieved in the output layer, it can be switched into the back propagation of error signal.

(2) Back propagation of error signal: the difference between the real output and expected output of the network is defined as the error signal; in the back propagation of error signal, the error signal is propagated from the output end to the input layer in a layer-by-layer manner. During the back propagation of error signal, the weighted value of network is regulated by the error feedback. The continuous modification of weighted value and offset value is applied to make the real output of network closer to the expected one.

\section{Results and discussion}

This study uses Artificial Neural Back-Propagation Network to predict the remote process controller (RPC) transferring pressure of the Novellus Vector equipment. The data is analysed from two different levels, including: the length of a Network training period and slack and peak season. The mean square error (MSE) was separately calculated for each category.

\subsection{Back-propagation network parameters}

The setup of network parameters in an Artificial Neural Network includes learning trials, learning rate, and the momentum correction coefficient. By setting up the learning rate at 0.1-0.3 for dynamic change, the momentum correction coefficient at 0.01-0.02, the specific setup values differ according to the network convergence status. When the MSE of the learning trail diminishes by 10-3/3000 epochs, this means the network learning process is completed. An average rate would be around 1,000-3,000 to achieve such standards.

Since the application of forward selection procedure will change the number of neurons in the hidden layer which leads to different groups of combinatorial optimization, this application should determine the number of neurons in the hidden layer prior to the selection process to prevent the process from being overly complicated.

To decide the number of neurons in the hidden layer, we should take the previously selected variable and substitute it into the network for training. Network training requires the exactly same setup, which includes learning trials, learning rate, and the momentum correction coefficient for a final decision. In this study, the number of neurons in the hidden layer is tested by setting the range at 1-2 times of the number of layers in the network. For example, if there were 10 input variables, then the number of layers would be 10 . This research tests 1 to 20 number of neurons in the hidden layer by achieving the optimal result for the forward selection procedure that follows. 


\subsection{Network training in peak and slack seasons}

Semiconductor industry has peak and slack seasons that change in cycle according to the time of the year. Right after Chinese New Year as often, the industry tends to be in the slack season since most of the clients have stocked up during the Christmas holidays already and do not need new supplies yet. Therefore, January and February are the slackest periods in the semiconductor industry. In contrast, when the clients use up their stock around March and April, the semiconductor industry starts to summit, and with May and June being the peak season. The months August to October are also another summit due to the client's need to stock up for the Christmas holidays.

(1) Slack season network training:

1,683 records of data were collected from the Transfer Pressure of the Novellus Vector from January 1st, 2010 to March 31st. The tested data retrieved from April 1st 2011 to April 5th consisted of 70 records. The learning times were set to 3,000, learning rate set to 0.1 , and the momentum correction coefficient set to 0. Since the training results of the Artificial Neural Back-Propagation Network do not always gain the same results, this study conducts the same experiment 10 times to ensure the network stability.

The results of the tests show that each round of the training and tests are slightly different. However, all the results converge within the 800th cycle. As shown in figure 3, the overall performance is fairly well with the $\mathrm{MSE}=0.0151$ and $\mathrm{RMSE}=0.6256$. Table 1 shows that by comparing the Mable Train-R data and Target data and by depicting the network training prediction output figure. Figure 2 depicts the default mode and its outlier can be predicted.

Table 1. Network training and performance tests of slack season.

\begin{tabular}{|c|c|c|}
\hline Hidden neuron & train - MSE & train - R \\
\hline 1 & 0.0142 & 0.6414 \\
\hline 2 & 0.0146 & 0.6470 \\
\hline 3 & 0.0152 & 0.6472 \\
\hline 4 & 0.0146 & 0.6234 \\
\hline 5 & 0.0149 & 0.6223 \\
\hline 6 & 0.0157 & 0.6609 \\
\hline 7 & 0.0158 & 0.5474 \\
\hline 8 & 0.0153 & 0.6034 \\
\hline 9 & 0.0148 & 0.6097 \\
\hline 10 & 0.0155 & 0.6534 \\
\hline Average & 0.0151 & 0.6256 \\
\hline \multicolumn{2}{|c}{} \\
\hline
\end{tabular}

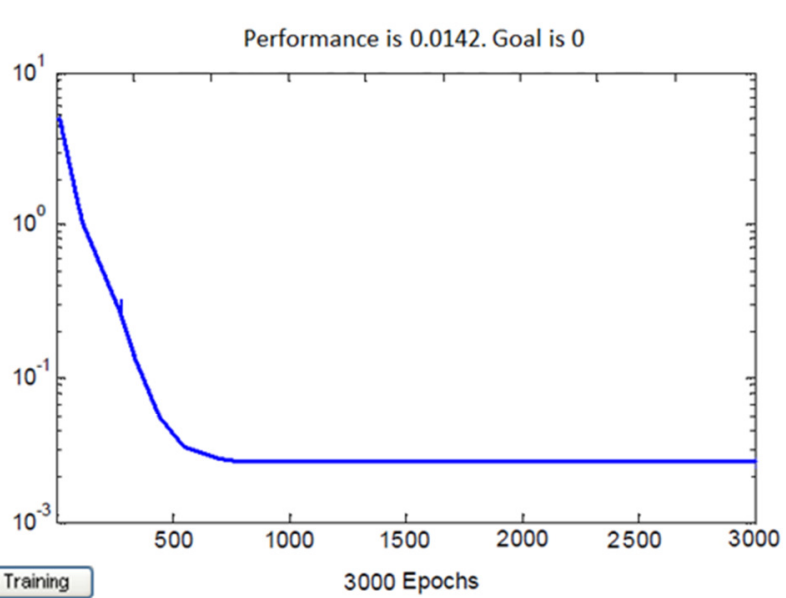

Figure 2. Network convergence figure of the slack seasons. 


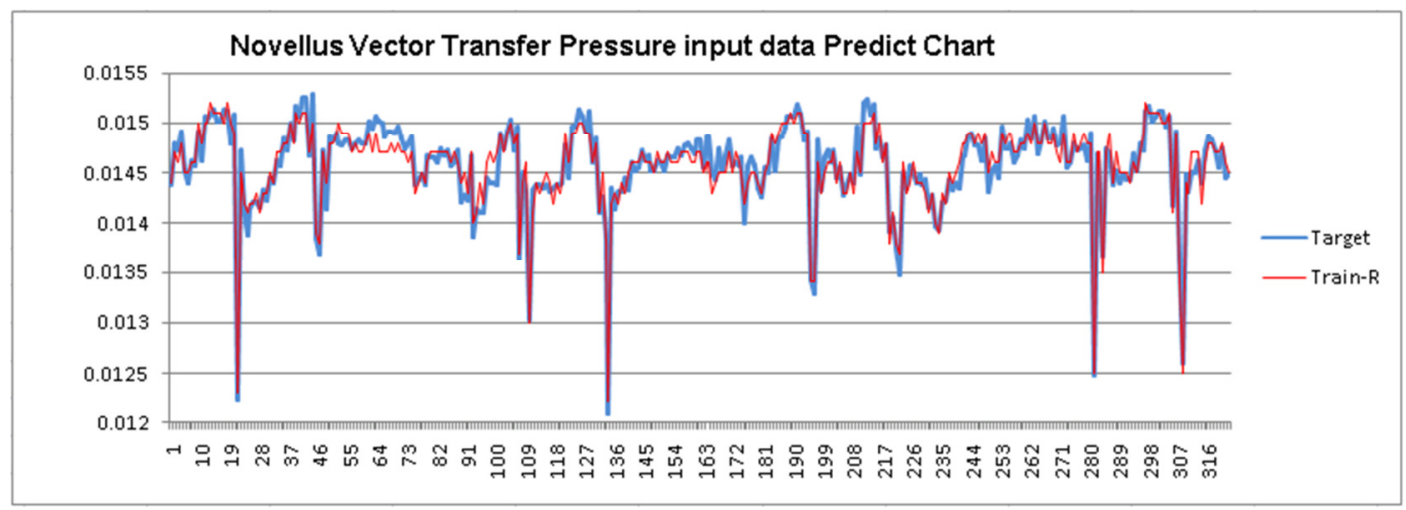

Figure 3. Network training prediction output figure of the slack seasons.

(2) Network training in the peak season

2,341 records of data were collected from the Transfer Pressure of the Novellus Vector from July 1st, 2010 to September 30th. The tested data retrieved from October 1st 2011 to October 5th consisted of 70 records. The learning times were set to 3,000, learning rate set to 0.1 , and the momentum correction coefficient set to 0. Since the training results of the Artificial Neural Back-Propagation Network do not always gain the same results, this study conducts the same experiment 10 times to ensure the network stability.

Table 2. Network training and performance tests of peak seasons.

\begin{tabular}{|c|c|c|}
\hline Hidden neuron & train - MSE & train - R \\
\hline 1 & 0.0119 & 0.6865 \\
\hline 2 & 0.0123 & 0.6925 \\
\hline 3 & 0.0129 & 0.6927 \\
\hline 4 & 0.0123 & 0.6673 \\
\hline 5 & 0.0126 & 0.6661 \\
\hline 6 & 0.0134 & 0.7073 \\
\hline 7 & 0.0135 & 0.5862 \\
\hline 8 & 0.0130 & 0.6460 \\
\hline 9 & 0.0125 & 0.6527 \\
\hline 10 & 0.0132 & 0.6993 \\
\hline Average & 0.0128 & 0.6696 \\
\hline
\end{tabular}

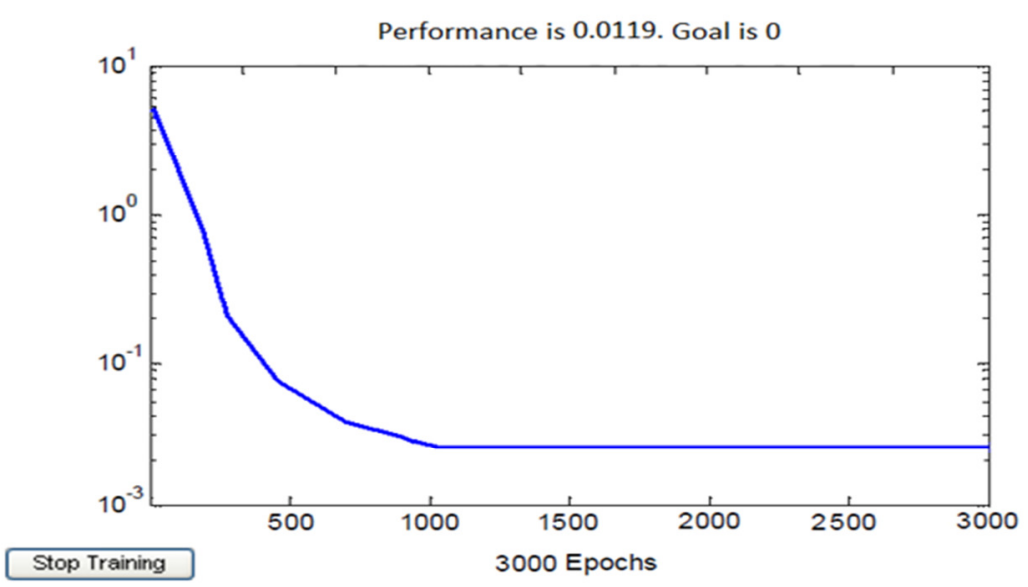

Figure 4. Network convergence figure of the peak seasons. 
The results of the tests show that each round of the training and tests are slightly different. However, all the results converge within the 1,050th cycle. As shown in figure 5, the overall performance is fairly well with the $\mathrm{MSE}=0.0128$ and $\mathrm{RMSE}=0$. 6696. Table 2 shows that by comparing the Mable Train- $\mathrm{R}$ data and Target data and by depicting the network training prediction output figure. Figure 4 depicts the default mode and its outlier can be predicted.

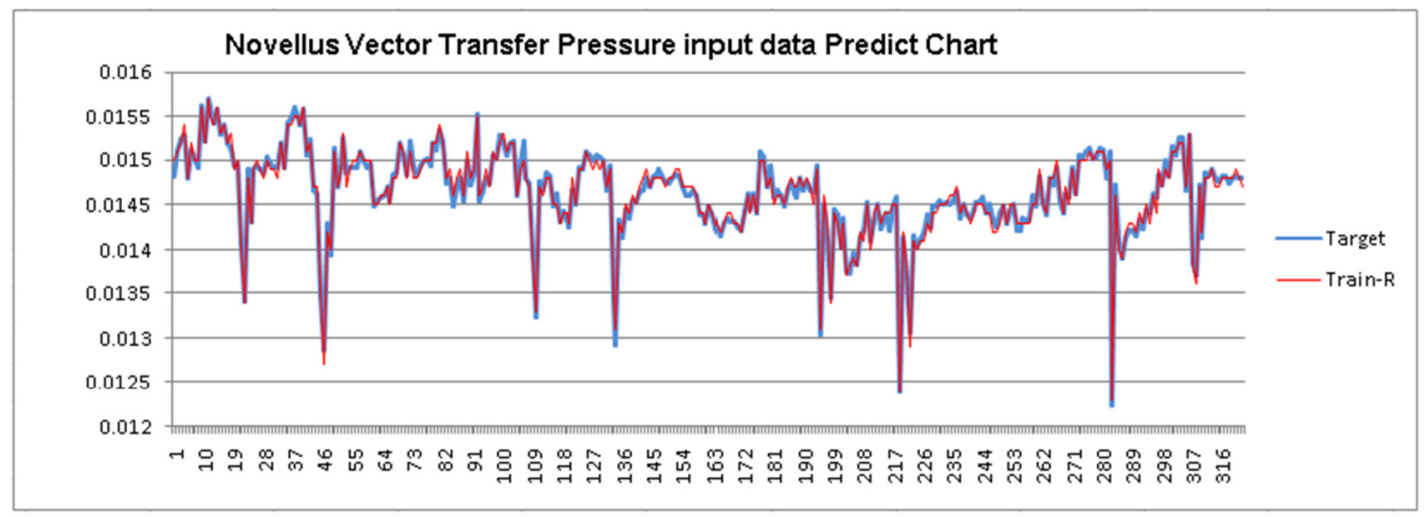

Figure 5. Network training prediction output figure of the peak seasons.

\section{Conclusion}

This study uses the mean square error (MSE) of the training output data to analyze if training results are acceptable and converged. The MSE equation can be seen in (1) shown below. The smaller MSE means the better network training results. In the network training process, the MSE might be unstable if the network is not converged. To improve such situation, the network learning rate and momentum correction coefficient should be readjusted to reach convergence.

This study analyzes the network training and prediction results by using the correlation coefficient (R) and MSE (equation (1) and (2) shown). The $\mathrm{n}$ in the equation signifies the total number of data inputs, $\mu$ notes the arithmetic mean, $\sigma$ notes the standard deviation, the subscript i notes the serial of data, subscript $t$ and a note the actual value and the network output value. This study analyzes the network output by calculating the correlation coefficient of the network trail output and the actual value and chooses the higher one as the optimal result. As shown in table 3, we take the trail network data and categorize it by network training time, low and high peak network training and calculate each the MSE of these data.

$$
\begin{aligned}
M S E & =\frac{1}{N} \sum_{i=1}^{n}\left(t_{i}-a_{i}\right)^{2} \\
R & =\frac{\left(\sum t_{i} a_{i}\right) n \mu_{t} \mu_{a}}{(n-1) \sigma_{t} \sigma_{a}}
\end{aligned}
$$

Table 3. The comparison of different types of training mode and performance tests.

\begin{tabular}{|l|c|c|}
\hline & Peak season & Slack season \\
\hline Train - MSE & 0.01279 & 0.01510 \\
\hline Train - R & 0.66965 & 0.62563 \\
\hline
\end{tabular}


The results of the network training found that the MSE of the peak season was smaller than that of slack one. Hence, we can conclude that peak and slack seasons will influence semiconductor machine outliers.

This study uses the Artificial Neural Back-Propagation Network model to detect the outliers in semiconductor machines. Due to the complicity of the process technology and many types of machines in the semiconductor industry, we chose the machine, Novellus Vector, which had the hardest gas control and pressure control for the network training. This study focuses on semiconductor factories as our research subjects. The results are based upon these factories. We suggest that future studies choose a different industry to conduct their study and also compare the differences between industries.

\section{Acknowledgement}

This paper was partially support by Ministry of Science and Technology, Taiwan. Grant No. 1022815-C-146-003-H and No. 104-2410-H-146-001 -

\section{References}

1. J.P. Card, M. Naimo, and W. Ziminsky, Run-to-run process control of a plasma etch process with neural network modelling, Qual Reliab Eng. Int., 14 (4), 247-260 (1998)

2. F.C. Chen, Back-propagation neural networks for nonlinear self-tuning adaptive control, IEEE Contr Syst Mag, 10 (3), 44-48 (1990)

3. W.C. Chen, A.H. Lee, W.J. Deng, and K.Y. Liu, The implementation of neural network for semiconductor PECVD process, Expert Syst. Appl., 32 (4), 1148-1153 (2007)

4. J.E. Dayhoff and J.M.D. Leo, Artificial neural networks, Cancer, 91 (S8), 1615-1635 (2001)

5. C.M. Fan, R.S. Guo, S.C. Chang, and C.S. Wei, SHEWMA: an end-of-line SPC scheme using wafer acceptance test data, IEEE T Semi Conduct M,13 (3), 344-358 (2000)

6. A. Goh, Back-propagation neural networks for modelling complex systems, Artif Intell Med, 9 (3), 143-151 (1995)

7. H.C. Pu and Y.T. Hung, Use of artificial neural networks: Predicting trickling filter performance in a municipal wastewater treatment plant, Envion Manage, 6, 16-27 (1995)

8. C.D. Himmel and G.S. May, Advantages of plasma etch modelling using neural networks over statistical techniques, IEEE T Semi Conduct M, 6 (2), 103-111 (1993)

9. S.J. Hong, W.Y. Lim, T. Cheong, and G.S. May, Fault detection and classification in plasma etch equipment for semiconductor manufacturing e-Diagnostics, IEEE T Semi Conduct M, 25 (1), 8393 (2012)

10. SC-IQ: Semiconductor Intelligence, Semiconductors down $2.7 \%$ in '12, may grow $7.5 \%$ in '13., http://www.semiconductorintelligence.com/?p=756 (2013)

11. G.S. May and C.J. Spanos, Fundamentals of Semiconductor Manufacturing and Process Control, John Wiley \& Sons (2006)

12. K. Mehrotra, C.K. Mohan, and S. Ranka, Artificial Neural Networks, the MIT Press (1997)

13. MIC. Output of Taiwan's Semiconductor Industry to Reach Approx. US\$35 Billion in 2009, Says MIC. from http://web.iii.org.tw/News/news_more?id=11, Accessed 03 March 2015 (2009)

14. J. Li, J. Cheng, J. Shi, and F. Huang, Brief introduction of back propagation (BP) neural network algorithm and its improvement, Advances in Computer Science and Information Engineering, 553-558 (2012)

15. B.S. de Ugarte, A. Artiba, and R. Pellerin, Manufacturing execution system-a literature review, Prod Plan Control, 20 (6), 525-539 (2009)

16. Y.E. Shao, C.J. Lu and C.C. Chiu, A fault detection system for an autocorrelated process using SPC/EPC/ANN and SPC/EPC/SVM schemes, Int. J. Innov. Comput. Int., 7 (9), 5417-5428 (2011)

17. G. Smith, Statistical Process Control and Quality Improvement: Prentice Hall, 576 (1998) 
18. J.Z. Wu, Inventory write-down prediction for semiconductor manufacturing considering inventory age, accounting principle and product structure with real settings, Computers \& Industrial Engineering, 65 (1), 128-136 (2011)

19. H.H. Yue, S.J. Qin, R.J. Markle, C. Nauert, and M. Gatto, Fault detection of plasma etchers using optical emission spectra, IEEE T Semi Conduct M, 13 (3), 374-385 (2000) 